க. சிவமணி அ, *

அ தமிழ்த்துறை \& ஆய்வு மையம், அரசுக் கல்லூரி சித்தூர், பாலக்காடு-678104, இந்தியா.

\title{
Tolkappiyam in Comparative Perspective - Purapporul Venbamaalai Vetchi Thinai
}

\author{
K. Sivamani a, * \\ a Department of Tamil \& Research Center, Government College Chittur, Palakkad-678104, Tamil Nadu, India
}

* Corresponding Author: sivamaniks2010@gmail.com

Received: 29-03-2021

Revised: 07-01-2022

Accepted: $13-01-2022$

Published: 28-02-2022

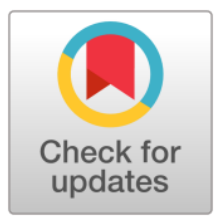

\begin{abstract}
This article compares grammatical descriptions of Tolkappiyar and Iyanarithanaar about Vetchi Thinai. It compares the descriptions about vetchi thinai and its Thuraigal told in Puraththinaiyiyal of Tholkappiyam with PurapporuL venbaa maalai. Vadavenkadam Tenkumari Aidai Tamilkorum Nallulakam Accepted Grammar Nool Tolkappiyam. Tolkappier, who divided semantics into writing and word, clearly defined dualistic grammar when defining his semantics. Due to the lack of internal and external departments, the external sector also became weak. They are Vetchi, Vanji, Uzhinai, Thumbai, Vagai, Kanchi, Padan. Among these, he has made a cut for kurinchi, a snare for the umbilical cord, a thump for weaving for medicine, a kanji for milk and a kanji for peruntinai. This article presents some of the similarities between the two departments found in both texts.
\end{abstract}

Keywords: Tolkappiyam, Tolkappiyar, Venbamaalai, Vetchi Thinai,

\section{முன்னுரை}

வடவேங்கடம் தென்குமரி ஆயிடைத் தமிழ்க்கூறு நல்லுலகம் ஏற்றுப் போற்றும் இலக்கண நூல் தொல்காப்பியம். எழுத்து, சொல் ஆகியவற்றுடன் பொருளிலக்கணத்தையும் வகுத்தளித்த தொல்காப்பியர் தனது பொருளதிகார இலக்கணத்தை வரையறுக்கையில் இருதிணைக்குமுரிய இலக்கணத்தைத் தெளிவாக வரையறுத்து விளக்கியுள்ளார் (Tolkappiyar, 2007; Sivalingan, 1995). அகத்திணை ஏழற்கும் புறமான திணையாதலால் புறத்திணையும் ஏழாயிற்று. அவையாவன: வெட்சி, வஞ்சி, உழிஞை, தும்பை, வாகை, காஞ்சி, பாடாண் என்பனவாகும். இவற்றில் குறிஞ்சிக்கு வெட்சியும், முல்லைக்கு வஞ்சியும், மருதத்துக்கு உழிஞையும் நெய்தலுக்குத் தும்பையும் பாலைக்கு வாகையும் பெருந்திணைக்குக் காஞ்சியும் கைக்களைக்குப் பாடாணும் புறமாக அமைத்துள்ளார். இவ்விரு நூல்களிலும் காணப்படும் வெட்சித் திணை குறித்த ஒப்புமைக்கூறுகளில் சிலவற்றை இக்கட்டுரை முன்வைக்கிறது.

\section{வெட்சி - தொல்காப்பியம்}

வெட்சித் திணையின் இலக்கணத்தை 
"வேந்துவிடு முனைஞர் வேற்றுப்புலக் களவின்

ஆதந் தோம்பல் மேவற்றாகும்.

என எடுத்துரைக்கும் தொல்காப்பியர், இது அரசனால் ஏவப்பட்டுப் பகைப்புலத்தில் காத்திருந்த மறவர்கள் பகைவரது நாட்டிற்குச் சென்று அந்நாட்டில் தனது நாட்டில் உள்ள ஆநிரைகளைக் காப்பவருக்குத் தெரியாமல் அவற்றைக் கவர்ந்து வந்து பாதுகாத்தலைக் குறிக்கும் என எடுத்துரைத்துள்ளார் (Vellaivaarananaar, 1983). அத்துடன் பசுக்களைக் கவர்ந்து வரும் வேளையில் பசுக்களுக்கு உரிமை உடையவர்கள் நிரையை மீட்டுச் செல்லுதலாகிய ஆநிரைகளை மீட்கும் செயலையும் வெட்சி என்றே குறிப்பார். வெட்சித் திணையாவது போர் குறித்த வேந்தர் இருவருள் ஒருவன் யுத்தத்தின் தொடக்கமாகப் பகைவர்களூடைய பசுக்களைக் கவர்வதும் அவன் கவர்ந்தவற்றை உரிய அரசன் மீட்பதுமாகும். இப்போர் தனிமாந்தர் வீரத்தைப் போற்றக்கூடியதாகும் (Ilampuranar, 1971).

தொல்காப்பியத்துள் குறிஞ்சி முதலாய ஐந்திணையும் கைக்கிளை, பெருந்திணையுஞ் சேர்ந்து எழுதிணையாக்கி அகப்பொருள் விளக்கப்பட்டாற்போல புறப்பொருளும் வெட்சி, வஞ்சி, உழிஞை, தும்பை, வாகை, காஞ்சி, பாடாண் என ஏழு திணைகளாக வகுத்து விளக்கப்படுகின்றது. இதனை, "ஆசிரியர் அகத்திணையேழுக்கும் இனமாக வகுத்துக்காட்டிய புறத்திணைகள் ஏழுமே பொருத்தமாய் நிற்றல் நன்கு விளங்குதலின், அவர்க்குப் பின்னெழுந்த பன்னிரு படலமுடையாரும், புறப்பொருள் வெண்பாமாலையாசிரியரும் அகத்திணை எழுக்கும் இனமாகும் புறத்திணைகள் பன்னிரண்டாமென்றது அத்துணைப் பொருந்துவதன்று" எனச் சுட்டிக்காட்டுதல் நோக்கத்தக்கதாகும் (llankumaranar, 2018).

வெட்சித் திணையில் படையியங்கு அரவம் முதல் கொடை ஈறாகப் பதினான்கு துறைகளைத் தொல்காப்பியர் வகுத்துள்ளார். மேலும்,

வெட்சியின் விரிவாக இருபத்தொரு துறைகளைக் கூறுகிறார். அவற்றில் முதல் பதின்மூன்று துறைகள் ஆநிரைகளைக் கவர்தலைக் குறிக்கவும் அடுத்து வரும் எட்டுத் துறைகள் ஆநிரைகளை மீட்டு வருதலைக் குறிப்பதாகவும் பாகுபாடு செய்துள்ளார். நிரை கொள்வோரும் நிரை மீட்போரும் வெட்சி மறவர் எனப்படுவர். இவர் வெட்சிப் பூவைச் சூடுவர்.

ஆதந்து ஓம்பல் என்றதனால் களவில் ஒருவன் கைப்பற்றிக் கொண்டு வந்த நிரையை அந்நிரைகளுக்கு உரியவர் மீட்டு ஓம்புவரென்றும் பொருள் தருவதால் அவ்வவ் வேந்தர்தம் தண்டத் தலைவர்கள் நிரை கோடற்கும் உரியவர்களாதலால் கோடற்றொழில் இருவருக்கும் உண்டு. எனவே அடித்துக் கோடலும் மீட்டுக் கோறலும் வெட்சியாயிற்று.

மீட்டலைக் கரந்தை எனக் கூறுவாறுமுளர். ஆனால் தொல்காப்பியர் வெட்சி, கரந்தை இரண்டையும் வெட்சி என்றே கொள்வார். இதனை,

"அகத்திணை மருங்கின் அரில்தப வுணர்ந்தோர்

புறத்திணையிலக்கணந் திறப்படக் கிளப்பின்

வெட்சிதானே குறிஞ்சியது புறனே

உட்குவரத் தோன்றும் ஈரேழ் துறைத்தே"

என்னும் தொல்காப்பிய நூற்பா எடுத்துரைக்கும் (Vellaivaarananaar, 1983).

"படையியங்கரவம் முதலாக எண்ணப்பட்ட பதினான்கு துறைகளும் வெட்சிசூடிப் பகைப்புலத்து ஆநிரைகளைக் கவர்வார்க்கே உரியனவாகக் கொண்டார் இளம்பூரணர்." வந்த ஈரேழ் வகையிற்றாகும்" என்பதனை ஈரேழ் வந்த வகையிற்றாகும் என இயைத்து, இவை பதினான்கும் நிரை கவர்வார்க்கும் நிரை மீட்பார்க்கும் உரியவாய் இருவகைப்பட்டு இருபத்தெட்டாம் எனக் கொண்டார் நச்சினார்க்கினியர். இது தொல்காப்பியனார் கருத்தன்று. எனினும் இப்பதினான்கு துறைகளும் நிரை கவர்வார்க்கும் நிரைமீட்பார்க்கும் ஒப்பு உரியனவாகப் பிற்காலத்தில் இயற்றப்பட்ட பெரும் பொருள் விளக்கம் என்ற புறத்திணை இலக்கண இலக்கிய நூலில் இடம்பெற்றிருத்தலால், இலக்கியம் கண்டற்கு இலக்கணம் இயம்பல் என்னும் முறைமை பற்றி 'ஒன்று இரண்டாய் இருபத்தெட்டாயிற்று என நச்சினார்க்கினியர் 
பொருள் வரைந்து எடுத்துக்காட்டுத் தந்து விளக்கியுள்ளமை பொருத்த முடையதேயாகும்" என வெள்ளைவாரணனார் குறிப்பிடும் விளக்கம் பொருத்தமுடையதாகும் (Vellaivaarananaar, 1983).

\section{புறப்பொருள் வெண்பாமாலை}

பன்னிரு படலம் என்னும் பழைய புறப்பொருள் இலக்கண நூலின் வழி நூல் புறப்பொருள் வெண்பாமாலை. இச்செய்தியைத் தொல்காப்பிய மரபியலில் ஒரு நூற்பாவுக்கு உரை எழுதுகையில் பேராசிரியர் எடுத்துக் கூறியுள்ளார்.

புறப்பொருள் வெண்பாமாலையில் வெட்சித் திணையும் கரந்தைத்திணையும் தம்முள் மறுதலைத் திணைகளாகும். வெட்சி, கரந்தை ஆகிய இரு திணைகளையும் வெவ்வேறு திணைகளாகப் புறப்பொருள் ெண்பாமாலை குறிப்பிடுகிறது. பன்னிரண்டு திணைகளுடன் ஒழிபு என ஒரு பகுதியையும் இந்நூல் கொண்டுள்ளது.

முதனூலோடு சிறிது வேற்றுமைப்பட நூல் செய்தல் மரபு என்பதால் தொல்காப்பியர் தமது நூலில் குறிப்பிட்டுள்ள திணை, துறை எண்ணிக்கையில் தமது சமகால இலக்கிய, இலக்கண மரபோடு ஒத்துப்போகும் வகையில் சிற்சில மாற்றங்களைச் செய்து ஐயனாரிதனார் தமது நூலைப் படைத்துள்ளதகாகக் கொள்ளலாம். ஐயனாரிதனார் வெட்சித் திணையை விளக்க இருபது துறைகளையும் கரந்தைத் திணையை விளக்கப் பதினான்கு துறைகளையும் வகுத்து விரிவாக விளக்கியுள்ளார். தொழில் வேறுபாட்டால் வெட்சி, கரந்தை எனத் திணைகளை வகுத்துக்கொண்டார் எனக் கொள்ளவும் இடமுண்டு.

\section{வெட்சித் திணையின் வகைகள்}

தொல்காப்பியத்திற்குப் பின்னர் தோன்றிய இலக்கண நூற்களாகிய வெட்சித் திணையினைப் புறப்பொருள் வெண்பா மாலை

“வென்றிவேந்தன் பணிப்பவும் பணிப்பின்றியும்

சென்றி கல்முனை ஆதந்தன்று"

என்ற நூற்பாவில் இரண்டு வகைகளாக எடுத்துரைத்துள்ளது (Ramasubramanian, 2009). போரைத் தொடங்கும் முன்பு பகைப்புலத்தே உள்ள பசுக்களைத் தனது ஓட்டிக்கொண்டு வந்து அவற்றைப் பாதுகாத்தலைச் செய்வான். அவ்வேளையில் அரசனது போர் வீரர்கள் அரசன் ஏவியபொழுதும் அரசன் ஏவாத பொழுது தனது சொந்த முயற்சியாலும் பசுக்களைக் கவர்ந்து தனது நாட்டிற்கு ஓட்டி வருவார்கள் என்பதை எடுத்துக் கூறியுள்ளன.

\section{பூவணிதல்}

போர் புரியும் அரசன் தாம் சூடியிருந்த பூவின் தன்மையைக்கொண்டு திணை வகுத்தனர். முற்காலத்துத் தமிழ் மக்கள் போர் முதலான புற ஒழுக்கங்கட்கும் அன்பு பற்றிய அகவொழுக்கங்கட்கும் ஓவ்வொரு பூவை அடையாளமாகக் கொண்டிருந்த செய்தி வெளியாதலால் அவர்க்குப் பூவணிவதிலிருந்த பெருவிருப்பம் புலனாவதாம்.

பண்டைத் தமிழ்த் தலைவருடைய போர் முறையானது நிரை கோடல், நிரை மீட்சி என்பவற்றை ஆரம்பமாகக் கொண்டு நிகழ்வதென்பது விளக்கமாகும். பாரத காலத்தே இரவில் நிரை கோடலும் மீட்டலும், யுத்த முறையின் தொடக்கமாக விளங்கிய செய்தி வெளியாகின்றது. வெட்சி என்பது சூடும் பூவாற் பெற்ற பெயர் என்பார் (Vellaivaarananaar, 1983). 


\section{கரந்தை}

வெட்சியார் ஆநிரைகளைக் கவர்ந்தமை கேட்ட அவ்வானிரையை உடைய அரசன் படைமறவர் விரைந்து வெட்சியாரோடே இடைவழியிற்பொருது தமது நிரையினை மீட்டது கரந்தைத் திணை எனப்படும். ஆநிரைகளை மீட்பதைக் கரந்தை எனத் தனித்திணையாகப் புறப்பொருள் வெண்பாமாலை கூறும். இக்கரந்தைத் திணை பதினான்கு துறைகளைக் கொண்டுள்ளது. இதன்படி இவ்வொழுக்கத்துக்குக் கரந்தைப் பூச் சூடுதல் உரித்தாம். வயல்களிலுள்ள கொட்டைக் கரந்தை என்னும் பூடே இக்கரந்தை என்பர். (பதிற் 40) இவ்வொழுக்கத்தை வெட்சித்திணையின்கட்பட்ட வழுவமைதியாக்கி ஆரமரோட்டல் முதலிய நான்கு துறைகளிலே அடக்கியுள்ளார் தொல்காப்பியர்.

\section{துறை வேறுபாடுகள்}

புறப்பொருள் வெண்பாமாலை கரந்தையொழுக்கத்துக்கு எடுத்துக்காட்டிய போர் மலைதல், வேத்தியன் மலிவு, பிள்ளைத் தெளிவு, பிள்ளைப் பெயர்ச்சி, என்னும் ஆறு துறைகளையும் தொல்காப்பியனார் அவ்வொழுக்கத்துக் காட்டிய ஆரமரோட்டல், வேந்தன் சீர்சால் சிறப்பு, தலைத்தாள் நெடுமொழி, வருதார்த் தாங்கல், வாள் வாய்த்துக் கவித்தல் பிள்ளையாட்டு என்னுந் துறைகளுள் முறையே அடக்கியுள்ளார்.

புறப்பொருள் வெண்பா மாலையில் அமைந்துள்ள புலனறி சிறப்பு, பிள்ளை வழக்கு ஆகிய துறைகள் தொல்காப்பியத்தில் அமையவில்லை. ஏனைய துறைகள் யாவும் தொல்காப்பிய வெட்சித் திணைத் துறைகளோடு பொருந்தி வருகின்றன.

\section{முடிவுரை}

இவ்வாறு தொல்காப்பியம் குறிப்பிடும் வெட்சித் திணை குறித்த கருத்துக்களும் பின்னாளில் தோன்றிய புறப்பொருள் வெண்பாமாலை இலக்கண நூலில் காணப்படும் வெட்சி, கரந்தை போன்ற திணை மற்றும் துறைகளில் காணலாகும் பண்டைத் தமிழரின் போர் முறை குறித்த செய்திகள் திணை, துறை எண்ணிக்கை வேறுபாடுகள், வெட்சித் திணையில் கூறப்படும் செய்திகளில் இவ்விருவருக்குமிடையே காணப்படும் வேறுபாடுகள், இவ்விரு இலக்கண நூல்களது காலகட்டத்தில் தோன்றிய இலக்கியங்களையொட்டி இலக்கணங்கள் உருவான தன்மை போன்ற பல்வேறு செய்திகள் இக்கட்டுரையில் கோடிட்டுக் காட்டப்பட்டுள்ளன.

\section{References}

Ilampuranar, (1971) Tolkappiyam Porulathikaram, Kazhaga veliyedu, Chennai, India.

Ilankumaranar, U.A., (2018) Tolkappiyam - Thelivurai, Fourth Tamil Sangam, Madurai, India.

Ramasubramanian, V, T., (2009) Puraporul Venbamalai, Meenakshi Puthaka Nilayam, Madurai, India.

Sivalingan, A., (1995) Tolkappiyam-Porulatikaram uraivalam, International Institute of Tamil Studies, Chennai, India.

Tolkappiyar, (2007) Tolkappiyam Sollathikaram, Saraswathi Mahal Library, Tanjore, India.

Vellaivaarananaar, K., (1983) Tholkappiyam puraththinaiyiyal uraivalam, Madurai Kamaraj University, Madurai, India.

Funding: No funding was received for conducting this study.

Conflict of Interest: The Author has no conflicts of interest to declare that they are relevant to the content of this article. 
About the License:

\section{(c)(1)}

Attribution 4.0 International (CC BY 4.0)
(C) The Author 2022. The text of this article is licensed under a Creative Commons Attribution 4.0 International License 